\title{
Accuracy of Clinical Evaluation of Patients with Site-specific Vaginal Wall Prolapse and Its Corelation to Surgical Diagnosis
}

\author{
${ }^{1}$ Garima Sharma, ${ }^{2}$ Shashank V Parulekar
}

\begin{abstract}
Introduction: Pelvic organ prolapse (POP) is a defect of a specific vaginal segment characterized by descent of the vagina and associated pelvic organ. An understanding of normal pelvic support structures provides the basis for the identification of the defect and anatomic approach to repair.
\end{abstract}

\section{Aims and objectives:}

- To clinically evaluate the patients of uterovaginal prolapse for site-specific defects and levels of damage.

- To corelate the clinical evaluation with evaluation during surgery.

- To perform the site-specific repair of the defects.

Materials and methods: A prospective observational study was conducted on 45 women admitted to the gynecological ward of King Edward Memorial (KEM) Hospital, Mumbai, India with symptoms of POP during a period of 13 months after seeking ethical clearance. After obtaining written informed consent, patients were subjected to detailed clinical evaluation for the type of prolapse, degree, and the site-specific defect. Patients were taken for surgery. Identification of the site-specific defect followed by restoration of the anatomic relationships by either repairing connective tissue supports or by creating a compensatory support mechanism was done.

Results: On analysis of our study, it was observed that there is mild agreement $(0.4-0.6)$ between the clinical and operative diagnosis of transverse defect and unilateral paravaginal defect of anterior compartment. However, there is good agreement $(0.6-0.8)$ between the clinical and operative diagnosis of bilateral paravaginal defects of anterior compartment. The clinical diagnosis for transverse defect of posterior compartment has accuracy of $88.2 \%$

Conclusion: The intraoperative diagnosis of site-specific defects remains the gold standard for detection of these defects.

Keywords: Arcus tendineus fasciae pelvis, Arcus tendineus fasciae rectovaginalis, Paravaginal defect, Pelvic organ prolapse, Prospective observational study, Site-specific defect, Transverse defect.

\footnotetext{
${ }^{1}$ Consultant, ${ }^{2}$ Professor and Head

1,2Department of Obstetrics and Gynecology, Seth Gordhandas Sunderdas Medical College and King Edward Memorial Hospital Mumbai, Maharashtra, India

Corresponding Author: Garima Sharma, Consultant Department of Obstetrics and Gynecology, Seth Gordhandas Sunderdas Medical College and King Edward Memorial Hospital Mumbai, Maharashtra, India, Phone: +918767586092, e-mail: garima23sharma@gmail.com
}

How to cite this article: Sharma G, Parulekar SV. Accuracy of Clinical Evaluation of Patients with Site-specific Vaginal Wall Prolapse and Its Corelation to Surgical Diagnosis. J South Asian Feder Obst Gynae 2018;10(3):175-181.

Source of support: Nil

Conflict of interest: None

Date of received: 06/18/2017

Date of acceptance: 04/16/2018

Date of publication: December 2018

\section{INTRODUCTION}

Pelvic organ prolapse occurs when abnormal descent or herniation of the pelvic organs occurs from their normal attachment sites or their normal position in the pelvis. The pelvic structure that may be involved include the uterus (uterine prolapse) or vaginal apex (apical vaginal prolapse), anterior vagina (cystocele), or posterior vagina (rectocele). Pelvic organ prolapse is a defect of a specific vaginal segment characterized by descent of the vagina and associated pelvic organ. ${ }^{1}$ It affects approximately half of all postmenopausal women. ${ }^{2}$

Patients may present with varying degrees of prolapse. In complete POP, multiple defects are present in the anterior, lateral, posterior, and apical compartments. ${ }^{1}$ In addition to vaginal birth, aggravating factors like postmenopausal atrophy, obesity, hysterectomy, chronic chest conditions, and constipation also contribute to its development.

Prolapse is associated with significant morbidity or deterioration of lifestyle; however, the mortality from this condition is negligible. One-third of all hysterectomies in postmenopausal women and $81 \%$ of vaginal hysterectomies are performed for prolapse. ${ }^{3}$

\section{Anatomical Considerations}

An understanding of normal pelvic support structures provides the basis for the anatomic approach to repair. The perineum, rectovaginal septum, pericervical ring, uterosacral ligaments, and presacral periosteum form the vaginal suspensory axis.

The basic concepts that must be satisfied to complete a prolapse surgery are proximal vaginal suspension, 
mid-vaginal lateral attachment, and distal vaginal fusion to the perineum and urogenital fascia. ${ }^{4,5}$

Full-length and full-width dissection followed by meticulous repair is required to achieve these goals. The most important key to successful repair of the anterior compartment defect is the ability to visually distinguish between visceral fascia of the bladder wall and the pubocervical septum.

The important supportive components of the pelvic floor are pelvic diaphragm and endopelvic fascia. ${ }^{6}$

The levator ani muscles are the most important muscles in the pelvic floor. The normal levators maintain a constant state of contraction. As a result, these muscles provide an active floor that supports the weight of the abdominopelvic contents against the forces of intraabdominal pressure. ${ }^{1}$

Two important landmarks, arcus tendineus levator ani and arcus tendineus fasciae pelvis or white lines, serve the function of mid-vaginal lateral support. Arcus tendineus fasciae rectovaginalis serves as the lateral support to the distal rectovaginal septum. ${ }^{4}$

Endopelvic fascia ${ }^{4}$ supports and invests all the midline organs and structures of the pelvis. It consists of three parts:

1. Parietal pelvic fascia

2. Visceral pelvic fascia

3. Deep endopelvic connective tissue

Deep endopelvic connective tissue is most important in the clinical applied anatomy of the pelvis. A thorough site-specific assessment for the identification of pelvic defects preoperatively as well as intraoperatively followed by repair of the defects will minimize the chance of recurrence of POP.

\section{Site-specific Vaginal Wall Defect}

\section{Anterior Compartment Defects ${ }^{7}$}

The anatomic components are cystocele and urethrocele. The anterior compartment defects are paravaginal, central midline, and central transverse defects. The most common pattern is right paravaginal defect and transverse proximal separation of the pubocervical septum from the pericervical ring.

\section{Posterior Compartment Defects ${ }^{8}$}

The anatomic components are rectocele and enterocele. Different types of site-specific defects are:

- Detachment of the rectovaginal septum from uterosacral/pericervical connective tissue ring

- Central or lateral defects in the mid-vaginal portion of rectovaginal septum

- Detachment of the rectovaginal septum from perineal body
- Disruption of the perineal body

- Disruption or attenuation of external anal sphincter

\section{AIMS AND OBJECTIVES}

- To clinically evaluate the patients of uterovaginal prolapse for site-specific defects and levels of damage

- To correlate the clinical evaluation with evaluation during surgery

- To perform the site-specific repair of the defects

\section{MATERIALS AND METHODS}

A prospective observational study was conducted on 45 women admitted to the gynecological ward of K.E.M. Hospital, Mumbai, India with symptoms of POP during a period of 13 months after seeking permission from the Institutional Ethics Committee.

\section{Inclusion Criteria}

- Women willing to participate in the study.

- Women with stage 1 symptomatic, stages 2, 3, and 4 vaginal prolapse, which cannot be treated with nonsurgical methods.

- Women without vaginal, cervical, and lower urinary tract infection.

- Women without genital malignancy.

\section{Exclusion Criteria}

- Women with stage 1 prolapse amenable to treatment with nonsurgical methods.

- Women not fit for surgery due to other high risk factors, such as thromboembolic risk, limited cardio and pulmonary function.

- Women who were pregnant or in puerperium or postabortal state.

An informed valid consent was taken from the patients and the relatives. A detailed history of the patient's complaints was taken with reference to:

- The duration of prolapse

- Bladder and bowel complaints

- Sexual history

- Medical history (especially history of chronic cough, connective tissue disorders, and smoking)

- Past surgical history

- Thorough general physical examination of the patient was done.

- Abdominal examination was performed to look for the abdominal tone and scars of previous surgeries.

- Patient was placed in lithotomy position and external genitalia were cleaned with antiseptic solution.

- Condition of external genitalia and type and degree of prolapse were noted on inspection. 
- Patient was asked to strain and the part which descends first was noted.

- Then, local examination was done to evaluate the site and type of prolapse.

- Paravaginal defects in anterior and posterior compartments were also noted.

\section{Anterior Compartment Defects}

An anterior vaginal wall retractor was used to replace the anterolateral sulci. If a paravaginal defect was present, replacement of the sulci caused the anterior vaginal wall to retract into a normal anatomic position and the bulge will not appear even on straining. The spatulas were removed in turn to determine whether paravaginal defect was unilateral or bilateral.

If a central bulge remained, it was reduced and supported by placing the anterior vaginal wall retractor transversely. Patient was asked to strain. Disappearance of the bulge indicated the presence of central transverse defect.

If the bulge still remained the two blades of the ring's forceps or sponge holding forceps were placed on each side of an imaginary line drawn between the urethra and the cervix and patient was asked to strain. The disappearance of the bulge indicated central midline defect.

The pattern of vaginal rugae on the vaginal epithelium and the condition of the lateral vaginal sulci were also noted. Vaginal epithelium was also inspected for atrophy created by the absence of estrogen.

\section{Posterior Compartment Defects}

Patient was asked to strain and the vaginal introitus and perineum were inspected. Anterior vaginal wall was elevated using blade of Sim's speculum. Posterior vaginal wall was inspected to note the presence/absence of rugae.

If bulge/descent was present, posterior vaginal fornix was supported toward the hollow of sacrum with the help of ring forceps/vaginal wall retractor and patient was asked to strain. If this maneuver reduced mass in total, it indicated detachment of rectovaginal septum from the uterosacral ligament, thereby producing the enterocele.

However, if the bulge persisted, it indicated rectocele. If the rectocele was reduced by placing the anterior vaginal wall retractor transversely, it indicated central break in the rectovaginal septum, thereby producing the central defect. If the bulge remained, the vaginal wall retractor was placed laterally on each side and patient was asked to cough. Disappearance of the bulge indicated lateral defect in the rectovaginal septum. Lateral defect indicated detachment of rectovaginal septum from levator fascia

The index finger was moved upward from the external anal sphincter toward vaginal introitus. If there was a defect at the attachment of the perineal body to the rectovaginal septum, the examining finger would enter the defect.

To elicit perineal descent, traction was applied downwards against the rectal side of the perineum. If the perineum was distensible and brought caudally with little or no resistance, it indicated perineal descent.

The perineal body was palpated with the thumb against the ipsilateral index finger in patient's rectum to note the amount of intervening tissue. After proper clinical evaluation and diagnosis of the type of defect, patient was taken for preoperative investigations and anesthetic evaluation. Patient was then taken for surgery after anesthetic fitness.

\section{Surgery}

Transvaginal approach was used for surgical repair of vaginal wall prolapse. Site-specific correction depended on access to all normal support structures. All patients received routine preoperative antibiotic prophylaxis at least 30 minutes prior to surgery. Patients were placed in the lithotomy position while they were under spinal/ epidural/general endotracheal anesthesia. A transurethral catheter was used to drain the bladder throughout the procedure.

\section{Site-specific Repair of Anterior Compartment}

The surgical goal was to identify the site-specific defect followed by complete restoration of pubocervical septum and of the attachments of the pericervical ring.

A transverse incision was made in the mucosa just above the portio vaginalis. Another incision was made in the midline starting from the transverse incision extending up to the lower limit of the cystocele, and still beyond if there was an associated urethrocele. The triangular flaps of the vaginal mucosa were raised by blunt and sharp dissection so that the bladder and urethra were completely freed. The plane of separation was continued laterally in both the directions to the lateral pelvic wall.

After the complete dissection, inspection was done for the fascial defects. The pubocervical fascia was differentiated from bladder fascia by its appearance. The pubocervical fascia was white and fibrous, while the visceral fascia of bladder was red and muscular. The edge of the detached pubocervical fascia was held with Allis' forceps. A transverse defect was confirmed if the approximation of the pubocervical fascia to the uterosacral ligament reduced the cystocele. If the approximation of the pubocervical fascia to the white line of one side reduced the cystocele, diagnosis of unilateral paravaginal defect was made. Similarly, the diagnosis of bilateral defects was made when the approximation of pubocervical fascia to the white line on either side reduced the bulge. 
The edges of the fascial septum were seen as separated from each other in the midline in case of central midline defect.

In case of lateral defects, the ischial spine on the side of the defect was palpated. The white line was identified close to it and then forward in its remaining extent. Simple interrupted sutures were taken with no. 1 to 0 polypropylene suture between the detached edge of the pubocervical septum and the arcus tendineus fascia pelvis. Placement of paravaginal suture began proximally in the area adjacent to the ischial spine and proceeded distally along the length of the fascia till the pubic tubercle. Contralateral side was checked for any iatrogenic defect.

In case of transverse defect, the lower edge of the fascial septum was held and sutured to the uterosacral/ cardinal ligament complex with interrupted sutures of no. 1 to 0 polypropylene. In midline central defect, the edges of the fascial septum separated from each other in the midline were sutured to each other with interrupted sutures of no. 1 to 0 polypropylene.

The excess of the vaginal mucosa was excised and the edges of the vaginal mucosa were approximated with continuous suture with chromic catgut 1 to 0 . The transverse incision in the vaginal mucosa was sutured similarly.

\section{Site-specific Repair of Posterior Compartment}

Identification of the site-specific defect followed by restoration of the anatomic relationships by either repairing connective tissue supports or by creating a compensatory support mechanism was the surgical goal.

The mucocutaneous border on each side was held with Allis' forceps at the points of junction of the posterior ends of the labia minora with the fourchette. An incision was made along the mucocutaneous border between the two Allis' forceps. A midline incision was made from the mucocutaneous junction to a point beyond the upper limit of rectocele. Triangular flaps of vaginal mucus membrane were elevated by sharp and blunt dissection on both the sides so that the rectum was freed.

After the dissection, detection of tears or detachment of the rectovaginal septum was done. Lateral defects were detachments of rectovaginal septum from the levator fascia; central defects were breaks in the rectovaginal septum.

- In case of detachment of the rectovaginal septum from the uterosacral ligaments, the uterosacral ligaments were identified and the edge of the detached rectovaginal septum was sutured to the ligaments with interrupted sutures of no. 1-0 polypropylene.

- In case of detachment of the rectovaginal septum from the perineal body, the edge of the detached rectovaginal septum was sutured to the perineal body with interrupted sutures of No. 1-0 polypropylene.
- In case of midline defect in the rectovaginal septum, the separated edges of the fascial septum in the midline were sutured to each other with interrupted sutures of no. 1 to 0 polypropylene.

After the repair of site-specific defects, the redundant vaginal mucosa was excised and the edges were approximated with no. 1 to 0 chromic catgut with continuous interlocking sutures. Perineal muscles were approximated with interrupted sutures with no. 1 to 0 chromic catgut. Perineal skin was sutured with interrupted sutures.

The statistical analysis of the results was done by:

- Kappa test

- Sensitivity, specificity, and likelihood ratios.

\section{RESULTS}

The study included women in the age group of 27 to 78 years, the mean age being 49.38 years. Majority of the women belonged to menopausal age group. Out of the 45 women, $28(62.2 \%)$ were menopausal, while 10 had regular menstrual cycles $(22.2 \%)$ and 7 had abnormal uterine bleeding $(15.6 \%)$.

Pelvic organ prolapse is more common in multiparous patients. The parity of the women ranged from 1 to 7 , with the majority, i.e., $28.9 \%$ having a parity of 3 and 4 respectively. Multiparity was the commonest risk factor seen in $75.5 \%$ of women followed by postmenopausal status in $62.2 \%$ and traumatic vaginal deliveries in $20 \%$. Other risk factors like chronic constipation, history of lifting heavy weight, and chronic cough were also noted.

The most common complaint was that of something coming out per vaginum, which was seen in 44 patients out of 45 (97.77\%). Out of 45 patients, 8 (17.77\%) complained of constipation, 6 (13.33\%) complained of dragging sensation, and 6 had complaints of lower back pain (13.33\%). Urinary complaints were present in 6 patients, out of which $5(11.11 \%)$ had complaint of frequency of micturition and 1 suffered from urgency. Two patients (4.44\%) had complaints of dyspareunia.

When classified into stages, $60 \%$ of patients had stage III, $28.9 \%$ had stage II, and $8.9 \%$ had stage IV of prolapse.

\section{Anterior Compartment Defects}

Single fascial defect was seen in $24(57.14 \%)$ patients, while multiple fascial defects were seen in 18 (40\%) patients. Transverse defect was diagnosed clinically in 42 patients (93.3\%). However, operative diagnosis was confirmed in 38 patients only $(84.4 \%)$. Out of the four patients who were clinically diagnosed to have transverse defect, one patient was intraoperatively diagnosed to have right paravaginal and other with left paravaginal defect. No defect was identified in remaining two due to marked atrophy of tissue. 
Table 1: Association among the cases between central transverse defect-clinical diagnosis and central transverse defect-operative diagnosis

\begin{tabular}{|c|c|c|c|c|}
\hline \multirow{2}{*}{$\begin{array}{l}\text { Central transverse } \\
\text { defect-clinical } \\
\text { diagnosis }\end{array}$} & & \multicolumn{2}{|c|}{$\begin{array}{c}\text { Central transverse defect- } \\
\text { operative diagnosis }\end{array}$} & \multirow[b]{2}{*}{ Total } \\
\hline & & $\begin{array}{l}\text { Cystocele-transverse } \\
\text { defect }\end{array}$ & No. & \\
\hline \multirow{2}{*}{$\begin{array}{l}\text { Cystocele } \\
\text { transverse defect }\end{array}$} & No. & 38 & 4 & 42 \\
\hline & $\%$ & 90.5 & 9.5 & 100.0 \\
\hline \multirow[t]{2}{*}{ No. } & No. & 0 & 3 & 3 \\
\hline & $\%$ & 0 & 100.0 & 100.0 \\
\hline \multirow[t]{2}{*}{ Total } & No. & 38 & 7 & 45 \\
\hline & $\%$ & 84.4 & 15.6 & 100.0 \\
\hline
\end{tabular}

Right paravaginal defect was diagnosed in 4 patients clinically. Out of these, two patients were diagnosed to have the defect intraoperatively. In other two patients, one had bilateral paravaginal defect and other did not have any paravaginal defect intraoperatively. Out of four patients having paravaginal defects intraoperatively, two were misdiagnosed clinically.

Left paravaginal defect was diagnosed intraoperatively in seven patients (15.6\%). Defect was diagnosed correctly in five patients on clinical examination. However, the defect was missed in 2 patients on clinical examination. One patient was diagnosed to have left paravaginal defect clinically but intraoperatively was found to have bilateral paravaginal defect.

Bilateral paravaginal defect was diagnosed on clinical examination in four patients (8.9\%). However, intraoperatively eight patients were diagnosed to have bilateral paravaginal defects (error of $9.8 \%$ ). The diagnosis was missed in four patients clinically.

When statistical tests of analysis were applied, it was observed that clinical diagnosis of transverse defect had accuracy of $90.5 \%$ with error of $9.5 \%$. Kappa value was 0.559 . This showed a mild agreement $(0.4-0.6)$ between the clinical and operative diagnosis. The sensitivity was $100 \%$ for the clinical diagnosis, while specificity was $12.8 \%$. Positive predictive value (PPV) was $90.48 \%$ and negative predictive value (NPV) was 100\%. Positive likelihood ratio was 1.75 (Tables 1 and 2).

Table 3: Association among the cases between unilateral paravaginal defects-clinical diagnosis/operative diagnosis

\begin{tabular}{|c|c|c|c|c|c|}
\hline \multirow{2}{*}{$\begin{array}{l}\text { Unilateral } \\
\text { paravaginal defect- } \\
\text { clinical diagnosis }\end{array}$} & & \multicolumn{3}{|c|}{$\begin{array}{c}\text { Unilateral paravaginal } \\
\text { defect-operative diagnosis }\end{array}$} & \multirow[b]{2}{*}{ Total } \\
\hline & & Right\# & Left & Other & \\
\hline \multirow[t]{2}{*}{ Right $^{\wedge}$} & No. & 2 & 0 & 2 & 4 \\
\hline & $\%$ & 50.0 & 0 & 50.0 & 100.0 \\
\hline \multirow[t]{2}{*}{ Left $^{\wedge}$} & No. & 0 & 5 & 1 & 6 \\
\hline & $\%$ & 0 & 83.3 & 16.7 & 100.0 \\
\hline \multirow[t]{2}{*}{ Other } & No. & 2 & 2 & 31 & 35 \\
\hline & $\%$ & 5.7 & 5.7 & 88.6 & 100.0 \\
\hline \multirow[t]{2}{*}{ Total } & No. & 4 & 7 & 34 & 45 \\
\hline & $\%$ & 8.9 & 15.6 & 75.6 & 100.0 \\
\hline
\end{tabular}

\#denotes unilateral paravaginal defect on operative diagnosis; ${ }^{\wedge}$ denotes unilateral paravaginal defect on clinical diagnosis
Table 2: Correlation between the clinical and operative diagnosis of central transverse defect of anterior compartment

\begin{tabular}{llll}
\hline & & \multicolumn{2}{c}{$\begin{array}{c}\text { 95\% Confidence } \\
\text { interval }\end{array}$} \\
\cline { 3 - 4 } Variables & Value & Lower & Upper \\
\hline Sensitivity & $100.00 \%$ & $90.75 \%$ & \\
Specificity & $42.86 \%$ & $9.90 \%$ & $81.59 \%$ \\
PPV & $90.48 \%$ & $77.38 \%$ & $97.34 \%$ \\
NPV & $100.00 \%$ & $29.24 \%$ & \\
Positive likelihood ratio & 1.75 & 0.92 & 3.32 \\
Negative likelihood ratio & 0 & & \\
\hline
\end{tabular}

Accuracy of clinical diagnosis for right paravaginal defect was $50 \%$ (error of 50\%), while for left paravaginal defect the accuracy was $83.3 \%$ (error of $16.7 \%$ ). Kappa value was 0.595 which showed a mild agreement $(0.4-0.6)$ between the clinical and operative diagnosis (Table 3).

Clinical diagnosis of presence of unilateral paravaginal defect irrespective of the side showed sensitivity of $63.64 \%$, specificity $91.18 \%$, PPV 70\%, NPV $88.57 \%$, positive likelihood ratio 7.21, and negative likelihood ratio 0.40 .

Bilateral paravaginal defects were diagnosed correctly on clinical examination in $100 \%$ cases. However, diagnosis of absence of defect was made accurately in $90.2 \%$ with error of $9.8 \%$. Kappa value for the above was 0.622 , which showed a good agreement (0.6-0.8) between clinical and operative diagnosis. The sensitivity of clinical diagnosis was $50 \%$, specificity $100 \%$, PPV $100 \%$, NPV $90.24 \%$, and negative likelihood ratio of 0.50 (Tables 4 and 5).

\section{Posterior Compartment Defects}

Posterior compartment defects were diagnosed in 34 patients $(75.55 \%)$. The most common defect diagnosed was transverse defect in 30 patients $(66.7 \%)$. Lax perineum was seen in 2 patients ( $4.44 \%$ ).

The transverse defect of rectocele was diagnosed clinically in 34 patients $(75.6 \%)$. However, the diagnosis was confirmed intraoperatively in only 30 patients (66.7\%). In remaining four, site-specific defect could not be defined in two patients due to atrophy of fascia. In other two, one was diagnosed to have right lateral defect and other had left lateral defect (Tables 6 and 7).

Table 4: Association among the cases between bilateral paravaginal defects-clinical diagnosis/operative diagnosis

\begin{tabular}{|c|c|c|c|c|}
\hline \multirow{2}{*}{$\begin{array}{l}\text { Bilateral paravaginal } \\
\text { defect-clinical } \\
\text { diagnosis }\end{array}$} & & \multicolumn{2}{|c|}{$\begin{array}{c}\text { Bilateral paravaginal } \\
\text { defect-operative } \\
\text { diagnosis }\end{array}$} & \multirow[b]{2}{*}{ Total } \\
\hline & & Yes & No & \\
\hline \multirow[t]{2}{*}{ Yes } & No. & 4 & 0 & 4 \\
\hline & $\%$ & 100.0 & 0 & 100.0 \\
\hline \multirow[t]{2}{*}{ No. } & No. & 4 & 37 & 41 \\
\hline & $\%$ & 9.8 & 90.2 & 100.0 \\
\hline \multirow[t]{2}{*}{ Total } & No. & 8 & 37 & 45 \\
\hline & $\%$ & 17.8 & 82.2 & 100.0 \\
\hline
\end{tabular}


Table 5: Correlation between the clinical and operative diagnosis of bilateral paravaginal defect of anterior compartment

\begin{tabular}{llll}
\hline & & \multicolumn{2}{c}{$95 \%$ Confidence interval } \\
\cline { 3 - 4 } Variables & Value & Lower & Upper \\
\hline Sensitivity & $50.00 \%$ & $15.70 \%$ & $84.30 \%$ \\
Specificity & $100.00 \%$ & $90.51 \%$ & \\
PPV & $100.00 \%$ & $39.76 \%$ & \\
NPV & $90.24 \%$ & $76.87 \%$ & $97.28 \%$ \\
Positive likelihood ratio & & & \\
Negative likelihood ratio & 0.50 & 0.25 & 1.00 \\
\hline
\end{tabular}

On analysis, the clinical diagnosis for transverse defect was accurate in $88.2 \%$, with error of $11.8 \%$. The clinical diagnosis for lax perineum showed $100 \%$ accuracy. The most common type of surgery performed was vaginal hysterectomy with site-specific repair in 30 patients.

Eight patients presented with immediate postoperative complications. Out of these, two had fever which responded to antipyretics, two had spinal headache which responded to analgesics, 3 complained of burning micturition which got relieved with hydration and alkalizers, and one patient had angina for which she was shifted to medicine ward. She responded to antianginal medications.

On 2-week follow-up, four patients presented with secondary hemorrhage and were treated with antibiotics. One patient had a wound gape, which healed with secondary intention.

\section{DISCUSSION}

Pelvic organ prolapse, though not a life-threatening condition, contributes significantly to a woman's quality of life. In our study, surgical corrections were done using a vaginal approach to perform site-specific endopelvic facial defect repair for the treatment of symptomatic POP, combined with hysterectomy and proximal uterosacral ligament suspension of the vaginal vault.

In the study by Awwad et al., ${ }^{9}$ it was observed that POP prevalence was $20.4 \%$ for women aged 20 to 29 years, $50.3 \%$ for women aged 30 to 39 years, $77.2 \%$ for women aged 40 to 49 years, and $74.6 \%$ for women aged 50 to 59 years. The incidence of POP roughly doubled with each decade in women aged 20 to 59 years in the pelvic organ support study (POSST). ${ }^{10}$ Physiologic aging, degenerative processes, and hypoestrogenism are associated with increased incidence. In our study, the mean age of women participating in the study was 49.38 years. The most common age group affected was 45 to 54 .

Increasing parity was associated with advancing prolapse in the POSST. ${ }^{10}$ In the reproductive risks for incontinence study at kaiser (RRISK study), Rortveit et $\mathrm{al}^{11}$ observed that the risk of prolapse increased significantly in women with one vaginal delivery [odds ratio (OR) 2.8], two (OR 4.1), or three or more (OR 5.3)
Table 6: Posterior compartment defect-clinical diagnosis

\begin{tabular}{lll}
\hline $\begin{array}{l}\text { Posterior compartment defect-clinical } \\
\text { diagnosis }\end{array}$ & No. & Percentage \\
\hline Rectocele-central transverse defect & 34 & 75.6 \\
Lax perineum & 2 & 4.4 \\
No. & 9 & 20.0 \\
\hline Total & 45 & 100.0 \\
\hline
\end{tabular}

deliveries compared with nulliparas. In our study, the parity of the women included ranged from 1 to 7 with the majority, i.e., $28.9 \%$ having a parity of 3 and 4 respectively.

In our study, among risk factors, multiparity was the commonest seen in $75.5 \%$ of women followed by postmenopausal status in $62.2 \%$ and traumatic vaginal deliveries in $20 \%$. Age, parity, and menopause were all independent factors for prolapse, while hormone replacement therapy, smoking, and body mass index were not, in the study on risk factors of POP by Kim et al. ${ }^{12}$

In the study by Miedel et al, ${ }^{13}$ it was observed that the feeling of vaginal bulge was specific to prolapse but not to any particular compartment. The association, however, was strongest with anterior wall prolapse. In our study, the most common complaint was that of something coming out per vaginum, which was seen in 44 patients out of $45(97.77 \%)$.

In a multicenter observational study of 1,004 women aged 18 to 83 years, at routine gynecological examination, $24 \%$ had stage 0 prolapse, $38 \%$ had stage $1,35 \%$ had stage 2 , and $2 \%$ had stage 3 prolapse according to POP questionnaire. ${ }^{14}$ However, in our study, when classified into stages, $60 \%$ of patients had stage $3,28.9 \%$ had stage 2 , and $8.9 \%$ had stage 4 of prolapse. The difference in the percentage is because of the different inclusion criteria for the present study.

\section{Anterior Compartment Defects}

Most common site-specific defect was transverse defect in $38(84.44 \%)$.

Barber et al ${ }^{15}$ have done a study to determine the accuracy of clinical assessment of paravaginal defects in women with anterior vaginal wall prolapse. The clinical assessment for paravaginal defects had a good sensitivity and NPV for left- and right-sided defects. However, the specificity and PPVs for the clinical assessment were poor. It was concluded that preoperative clinical assessment for

Table 7: Posterior compartment defect—operative diagnosis

\begin{tabular}{|c|c|c|}
\hline $\begin{array}{l}\text { Posterior compartment defect- } \\
\text { operative diagnosis }\end{array}$ & No. & Percentage \\
\hline Rectocele-central transverse defect & 30 & 66.7 \\
\hline Lax perineum & 2 & 4.4 \\
\hline Left lateral & 1 & 2.2 \\
\hline Right lateral & 1 & 2.2 \\
\hline No. & 11 & 24.4 \\
\hline Total & 45 & 100.0 \\
\hline
\end{tabular}


paravaginal defects cannot substitute for intraoperative assessment for fascial defects.

Segal et al. ${ }^{16}$ also performed a study to determine the prevalence of paravaginal defects and ascertain the correlation between diagnosing a paravaginal defect clinically and observing the presence of one intraoperatively. The study concluded that the overall prevalence of a paravaginal defect is low in patients with anterior vaginal wall prolapse. The standard clinical evaluation used to preoperatively detect a paravaginal defect is a poor predictor for the actual presence of a paravaginal defect and hence cannot replace the intraoperative assessment.

Physical examination should be followed by careful intraoperative assessment to diagnose paravaginal defects as this method has low specificity and PPV. Magnetic resonance imaging is an expensive alternative and may not be readily available. Paravaginal repair, both transvaginal and transabdominal approaches, is associated with low recurrence rates. ${ }^{17}$

\section{Posterior Compartment Defects}

Most common defect diagnosed was transverse defect in 30 patients $(66.7 \%)$. Lax perineum was seen in two patients $(4.44 \%)$. Two patients presented with vault prolapse. Sacrospinous vault suspension with site-specific repair was done for them. This shows that sometimes site-specific defect can be missed intraoperatively. Sitespecific defect identification and repair depends on the surgeon's expertise and understanding of the female pelvic anatomy.

Mean duration for the surgery was 2.36 hours. It depended on the operating surgeon's expertise and intraoperative complications.

\section{CONCLUSION}

On analysis of our study, it was observed that there is mild agreement (0.4-0.6) between the clinical and operative diagnosis of transverse defect and unilateral paravaginal defect of anterior compartment. However, there is good agreement $(0.6-0.8)$ between the clinical and operative diagnosis of bilateral paravaginal defects of anterior compartment. The clinical diagnosis for mere presence of posterior compartment defect has 100\% sensitivity but low specificity. The clinical diagnosis for transverse defect of posterior compartment has accuracy of $88.2 \%$.

The intraoperative diagnosis of site-specific defects remains the gold standard for detection of these defects. In order to prevent recurrence of POP, thorough understanding of the female pelvic anatomy is essential to correctly diagnose and manage these defects.

\section{REFERENCES}

1. Strohbehn K, Lazarou G, Grigorescu BA. Pelvic organ prolapse. Int Urogynaecol J 2006 Feb;17(2):160-164.

2. Swift SE. The distribution of pelvic organ support in a population of female subjects seen for routine gynecologic health care. Am J Obstet Gynecol 2000 Aug;183(2):277-285.

3. Allard P, Rochette L. The descriptive epidemiology of hysterectomy, province of Quebec, 1981-1988. Ann Epidemiol 1991 Nov;1(6):541-549.

4. ZimmermanCW.TeLinde'soperativegynecology.In:Section VII, Chapter 36A, Pelvic organ prolapse: basic principles. 10th ed. Wolters Kluwer; 2011. pp. 854-873.

5. DeLancey JO. Pelvic organ prolapse: clinical management and scientific foundations. Clin Obstet Gynecol 1993;36:895.

6. Moen MD. Surgery for urogenital prolapse. Rev Med Univ Navarra 2004;48(4):50-55.

7. ZimmermanCW.TeLinde'soperativegynecology.In:Section VII, Chapter 36B, Site-specific repair of cystouretherocele. 10th ed. Wolters Kluwer; 2011. pp. 874-881.

8. Julian Thomas M.TeLinde'soperativegynecology.In:Section VII, Chapter 362D, Posterior compartment defects.10th ed. Wolters Kluwer; 2011. pp. 892-910.

9. Awwad J, Sayegh R, Yeretzian J, Deeb ME. Prevalence, risk factors, and predictors of pelvic organ prolapse: a communitybased study. Menopause 2012 Nov;19(11):1235-1241.

10. Swift S, Woodman P, O'Boyle A, Kahn M, Valley M, Bland D, Wang W, Schaffer J. Pelvic Organ Support Study (POSST): the distribution, clinical definition, and epidemiologic condition of pelvic organ support defects. Am J Obstet Gynecol 2005 Mar;192(3):795-806.

11. RortveitG,BrownJS,ThomDH,VanDenEedenSK,CreasmanJM, Subak LL. Symptomatic pelvic organ prolapse: prevalence and risk factors in a population-based, racially diverse cohort. Obstet Gynecol 2007 Jun;109(6):1396-1403.

12. Kim S, Harvey MA, Johnston S. A review of the epidemiology and pathophysiology of pelvic floor dysfunction: do racial differences matter. J Obstet Gynaecol Can 2005 Mar;27(3):251-259.

13. Miedel A, Tegerstedt G, Maehle-Schmidt M, Nyrén O, Hammarström M. Symptoms and pelvic support defects in specific compartments. Obstet Gynecol 2008 Oct;112(4): 851-858.

14. Hall AF, Theofrastous JP, Cundiff GW, Harris RL, Hamilton LF, Swift SE, Bump RC. Interobserver and intraobserver reliability of the proposed International Continence Society, Society of Gynecologic Surgeons, and American Urogynecologic Society pelvic organ prolapse classification system. Am J Obstet Gynecol 1996 Dec;175(6):1467-1470.

15. Barber MD, Cundiff GW, Weidner AC, Coates KW, Bump RC, Addison WA. Accuracy of clinical assessment of paravaginal defects in women with anterior vaginal wall prolapse. Am J Obstet Gynecol 1999 Jul;181(1):87-90.

16. Segal JL, Vassallo BJ, Kleeman SD, Silva WA, Karram MM. Paravaginal defects: prevalence and accuracy of preoperative detection. Int Urogynecol J Pelvic Floor Dysfunct 2004 NovDec;15(6):378-383; discussion 383.

17. Nguyen JK. Current concepts in the diagnosis and surgical repair of anterior vaginal prolapse due to paravaginal defects. Obstet Gynecol Surv 2001 Apr;56(4):239-246. 